Article

\title{
Soil Organic Matter Accumulation and Carbon Fractions along a Moisture Gradient of Forest Soils
}

\author{
Ewa Błońska * and Jarosław Lasota \\ Department of Forest Soil Science, Faculty of Forestry, University of Agriculture, Al. 29 Listopada 46, \\ 31-425 Krakow, Poland; rllasota@cyf-kr.edu.pl \\ * Correspondence: eblonska@ar.krakow.pl
}

Received: 23 October 2017; Accepted: 14 November 2017; Published: 17 November 2017

\begin{abstract}
The aim of the study was to present effects of soil properties, especially moisture, on the quantity and quality of soil organic matter. The investigation was performed in the Czarna Rózga Reserve in Central Poland. Forty circular test areas were located in a regular grid of points $(100 \times 300 \mathrm{~m})$. Each plot was represented by one soil profile located at the plot's center. Sample plots were located in the area with Gleysols, Cambisols and Podzols with the water table from 0 to $100 \mathrm{~cm}$. In each soil sample, particle size, total carbon and nitrogen content, acidity, base cations content and fractions of soil organic matter were determined. The organic carbon stock (SOCs) was calculated based on its total content at particular genetic soil horizons. A Carbon Distribution Index (CDI) was calculated from the ratio of the carbon accumulation in organic horizons and the amount of organic carbon accumulation in the mineral horizons, up to $60 \mathrm{~cm}$. In the soils under study, in the temperate zone, moisture is an important factor in the accumulation of organic carbon in the soil. The highest accumulation of carbon was observed in soils of swampy variant, while the lowest was in the soils of moist variant. Large accumulation of $C$ in the soils with water table $80-100 \mathrm{~cm}$ results from the thick organic horizons that are characterized by lower organic matter decomposition and higher acidity. The proportion of carbon accumulation in the organic horizons to the total accumulation in the mineral horizons expresses the distribution of carbon accumulated in the soil profile, and is a measure of quality of the organic matter accumulated. Studies have confirmed the importance of moisture content in the formation of the fractional organic matter. With greater soil moisture, the ratio of humic to fulvic acids (HA/FA) decreases, which may suggest an increase in carbon mobility in soils.
\end{abstract}

Keywords: carbon distribution index; moisture gradient; soil organic matter fraction

\section{Introduction}

Accumulation of organic carbon in soils is currently discussed for many reasons. With the increased pool of carbon stored in terrestrial ecosystems, a reduction of carbon dioxide emissions into the atmosphere and prevention from climate changes are observed [1,2]. In the carbon cycle on Earth, soil is its most important reservoir. It is estimated that carbon contained in soil constitutes $75 \%$ of the total pool of organic carbon, exceeding twice the resources of carbon in the atmosphere [3]. The accumulation of organic carbon in forest soils depends on many factors, but predominantly on climatic conditions [4], soil properties [5], soil moisture [6,7], the type of plant cover and type of forest management [8-11]. The site conditions and plant coverage are strongly related to each other. Here, the site conditions play the main role via driving formation of certain species composition and the structure of plant cover, thus determining the type and direction of soil formation processes [12]. Among the physical properties of the soil, clay fraction content acts in the formation of soil organic matter (SOM) accumulation. Many authors have studied the influence of clay and silt content on the protection of SOM, and have found a relationship between the content of SOM and soil texture, mainly 
with the content of fine fractions (silt and clay) [13,14]. According to Wosten et al. [15], the accumulation and stabilization of soil carbon are associated with the content of clay; however, it may also depend on the soil moisture. Bauer et al. [16] and Meersmaus et al. [17] reported a strong influence of soil moisture on the decomposition of SOM, and consequently, on the carbon accumulation in the soil. SOM decomposition dynamics are influenced by $\mathrm{pH}$ making it an important variable, as an increase in SOM content is connected with changes of other soil parameters, namely $\mathrm{pH}$ [18].

By specifying the total content of soil carbon (C) [19] or by estimating the available fractions of SOM [20], the quantity and quality of SOM can be determined. To evaluate the changes in soil $\mathrm{C}$ dynamics due to agricultural use, the use of SOM chemical fractions was more effective than the determination of total SOM in Guimarães et al.'s [21] study. The SOM fraction can be used as an indicator of changes in the soil. The amount of SOM fractions reflects the potential mobility of $\mathrm{C}$ in the soil and the rate of SOM decomposition [22].

Until now, a limited number of studies reporting the relationship between the forest site condition, the accumulation of SOM, and fractions of humic substances has been published. There is a lack of studies on the effect of different moisture levels, soil properties, and natural forest plant communities on the quantity and quality of SOM in temperate climates. We hypothesize that: (1) soil moisture is an important factor in the accumulation of organic carbon in the temperate zone, and the highest accumulation of carbon was connected with a swampy variant of soils; (2) moisture has an effect on the proportion of carbon accumulation in the organic horizon to the accumulation in the mineral horizons; and (3) fractional composition of the organic matter reflects the rate of SOM decomposition in forest soils and depends on soil moisture.

\section{Materials and Methods}

\subsection{Study Area}

The study was performed in the Czarna Rózga Reserve in Central Poland (Figure 1). The reserve area is $185.6 \mathrm{ha}$, and within the limits of the reserve stands is the dominant species common alder (Alnus glutinosa), which are of natural origin. The silver fir (Abies alba), pedunculate oak (Quercus robur), common hornbeam (Carpinus betulus) and common ash (Fraxinus excelsior) play the role of admixtures. The study area is characterized by the following climatic conditions: the average annual rainfall is $649 \mathrm{~mm}$, the average daily temperature of the warmest month (July) is $17.8^{\circ} \mathrm{C}$ and the coldest month (January) is $-3.4^{\circ} \mathrm{C}$, and the length of the vegetation season lasts 200-210 days. Sample plots were located in the area with a predominance of fluvioglacial sand and loam with Gleysols, Cambisols and Podzols [23]. The groundwater level is the basic reason for differentiation of soil subtype and vegetations communities.

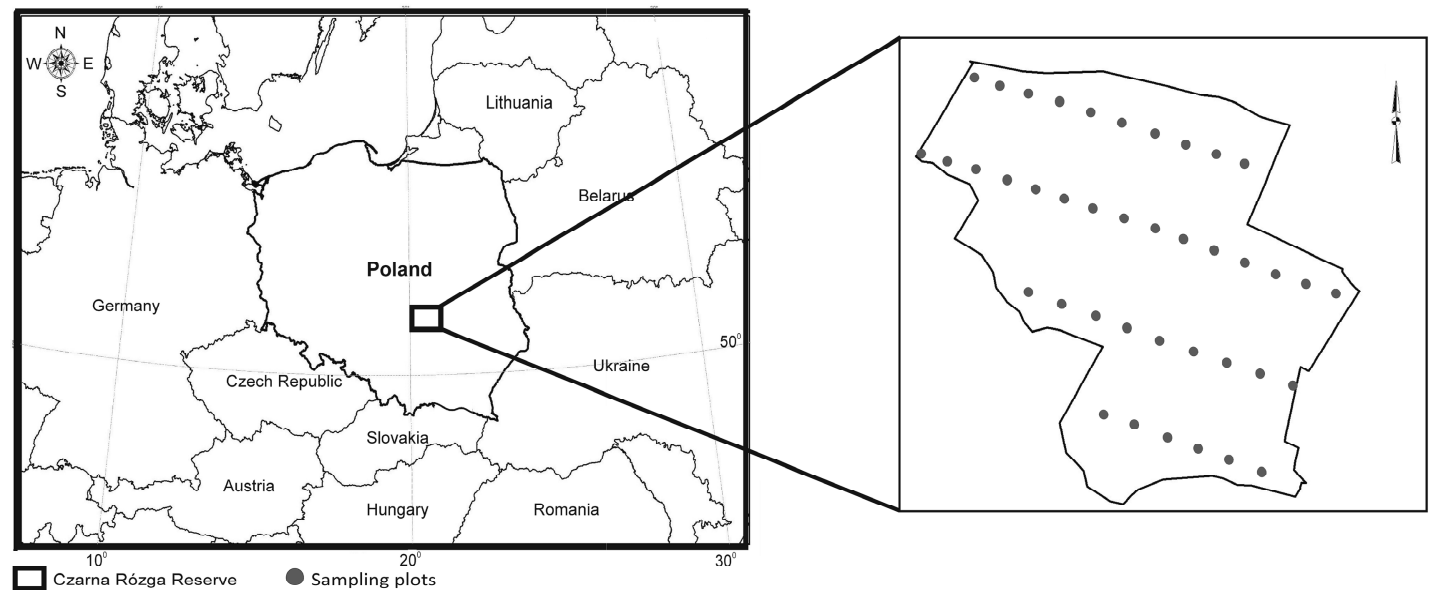

Figure 1. Study sites location (Czarna Rózga Reserve in Central Poland). 


\subsection{Sampling Scheme}

Forty circular test areas with a surface of 0.1 hectare were established within the area of the reserve. Test surfaces were located in a regular grid of points $(100 \times 300 \mathrm{~m})$ (Figure 1). Each plot was represented by one soil profile located at the plot's center. The surface horizons (organic- $\mathrm{O}$, mineral-A, or organic/mineral-AM) and the mineral topsoil (AE or AG) horizon were sampled. The subsoil gleyic horizon $(\mathrm{G})$ was sampled from the bottom of the topsoil horizon to $60 \mathrm{~cm}$ deep. The surface horizons' samples constituted a cumulative sample from five subsamples around the soil profile.

On each test surface (Table 1), site conditions including vegetation types, soil types, and moisture levels (based on the groundwater depth and using soil moisture monitoring sensors in the daily interval in May and June) were recorded. The obtained results revealed three types of vegetation communities (Abietetum albae, Tilio-Carpinetum ficarietosum and Fraxino-Alnetum) within the reserve and water table from 0 to $100 \mathrm{~cm}$ in soil profiles. The three variants of soil moisture content (first variant with water table $80-100 \mathrm{~cm}\left(\mathrm{WT}_{80-100}\right)$, second variant with water table $40-50 \mathrm{~cm}$-moist, and last variant with water table 0-30 $\mathrm{cm}$-swampy) were distinguished. In further analysis, the above sequence of soil moisture content $\left(\mathrm{WT}_{80-100}\right.$, moist and swampy) was considered. Ten areas that represent the Abietetum albae from Stagnosols and Podzols have been identified for the $\mathrm{WT}_{80-100}$ variant. The moist variant represents the Tilio-Carpinetum ficarietosum (19 areas). Gleysols dominated this variant. The third swampy variant (11 areas) is represented by the Fraxino-Alnetum dominated by Mollic Gleysols.

Table 1. Characteristic of vegetation and soils in groups of studied areas.

\begin{tabular}{|c|c|c|c|c|c|c|}
\hline $\begin{array}{l}\text { Moisture } \\
\text { Gradient }\end{array}$ & $\begin{array}{c}\text { Plant } \\
\text { Communities }\end{array}$ & $\begin{array}{l}\text { Dominant Stand } \\
\text { Species }\end{array}$ & $\begin{array}{l}\text { Dominant Ground } \\
\text { Cover Species }\end{array}$ & Type of Soil & $\begin{array}{l}\text { Depth of } \\
\text { Groundwater } \\
\text { Level (in the } \\
\text { Spring) (cm) }\end{array}$ & $\begin{array}{l}\text { Humus } \\
\text { Type }\end{array}$ \\
\hline $\mathrm{WT}_{80-100}$ & $\begin{array}{l}\text { Abietetum } \\
\text { albae }\end{array}$ & Silver fir & $\begin{array}{l}\text { Maianthemum bifolium } \\
\text { Luzula pilosa } \\
\text { Thiudium tamarescinum } \\
\text { Polytrichum attenuatum }\end{array}$ & $\begin{array}{l}\text { Podzols } \\
\text { Stagnosols }\end{array}$ & 80-100 & mor \\
\hline Moist & $\begin{array}{l}\text { Tilio-Carpinetum } \\
\text { ficarietosum }\end{array}$ & $\begin{array}{c}\text { Common } \\
\text { hornbeam, } \\
\text { Common alder }\end{array}$ & $\begin{array}{l}\text { Aegopodium podgraria } \\
\text { Galeobdolon luteum } \\
\text { Hepatica nobilis } \\
\text { Impatiens noli-tangere }\end{array}$ & Gleysols & $40-50$ & mull \\
\hline Swampy & Fraxino-Alnetum & Common alder & $\begin{array}{l}\text { Ficaria verna } \\
\text { Valeriana simplicifolia } \\
\text { Caltha palustris } \\
\text { Carex remota }\end{array}$ & $\begin{array}{l}\text { Mollic } \\
\text { Gleysols }\end{array}$ & $0-30$ & $\begin{array}{l}\text { mull } \\
\text { sticky }\end{array}$ \\
\hline
\end{tabular}

\subsection{Laboratory Analysis of Soil}

Soil samples obtained in the field were dried and sieved through $2.0 \mathrm{~mm}$ mesh. Using the potentiometric method, the $\mathrm{pH}$ of the samples was analyzed in $\mathrm{H}_{2} \mathrm{O}$ and $\mathrm{KCl}$. By using a laser diffraction technique (Analysette 22, Fritsch, Idar-Oberstein, Germany), the soil texture was determined. Carbon $\left(\mathrm{C}_{\mathrm{t}}\right)$ and nitrogen $(\mathrm{N})$ contents were measured with an elemental analyzer (LECO CNS TrueMac Analyzer) (Leco, St. Joseph, MI, USA). Using the method of Kononowa and Bielczikowa, fractional composition of humus was determined in which extraction is performed in a mixture of $0.1 \mathrm{M} \mathrm{NaOH}$ and $0.1 \mathrm{M} \mathrm{Na}_{4} \mathrm{P}_{2} \mathrm{O}_{7}$ [24]. In order to obtain a humin (Hm), humic acid (HA), and fulvic acid (FA) fraction, the chemical fraction was conducted. The relationships HA/FA and (HA+FA)/Hm were also calculated. Exchangeable $\mathrm{Ca}, \mathrm{Mg}, \mathrm{K}$ and $\mathrm{Na}$ were determined by inductively coupled plasma optical emission spectrometry (ICP-OES) (iCAP 6500 DUO, Thermo Fisher Scientific, Cambridge, UK) in $1 \mathrm{M}$ ammonium acetate at $\mathrm{pH} 7.0$ extracts. The sum of base cations (BC) and effective base saturation (BS) 
were calculated. Available phosphorus was determined by Bray-Kurtz's method. The hydrolytic acidity was determined in the samples after extracting $5 \mathrm{~g}$ of soil with $30 \mathrm{~mL} 1 \mathrm{~mol} \cdot \mathrm{L}^{-1}\left(\mathrm{CH}_{3} \mathrm{COO}\right){ }_{2} \mathrm{Ca}$ (shaking time $1 \mathrm{~h}$ ), followed by filtration. After extracting $5 \mathrm{~g}$ of soil with $30 \mathrm{~mL} \mathrm{KCl}$, exchangeable acidity was determined. Soil on the filter was washed several times by extractant solution, up to a volume of $200 \mathrm{~mL}$. About $25 \mathrm{~mL}$ of the obtained solution was titrated by potentiometric titration (automatic titrator, Mettler Toledo Inc., Columbus, $\mathrm{OH}$, USA) to a $\mathrm{pH}$ of 8.2. For determining the exchangeable aluminum and hydrogen, $\mathrm{NaF}$ was added to the $\mathrm{KCl}$ extract and titrated with $\mathrm{NaOH}$. Total cation exchange capacity (CECt) was calculated as a sum of base cations (BC) and hydrolytic acidity (Ah); and effective cation exchange capacity (CECe) was calculated as a sum of base cations (BC) and exchangeable acidity (Aex). Using samples with intact structure collected to metal cylinders, bulk density was determined via the drying-weighing method [25].

The soil organic carbon stock $\left(\mathrm{SOC}_{\mathrm{S}}\right)$ was calculated as a sum of its total content at particular soil horizons. In each of the analyzed soil samples, the SOC $S$ was calculated in the soil block area of $1 \mathrm{~m}^{2}$ and a depth from the surface to $60 \mathrm{~cm}$. The calculation of $S O C_{S}$ for the particular depths was made by summing the $\mathrm{SOC}_{\mathrm{S}}$ at subsequent genetic soil horizons according to the formula:

$$
\mathrm{SOC}_{\mathrm{S}}=\mathrm{C} \times \mathrm{D} \times \mathrm{m} / 10\left(\mathrm{Mg} \cdot \mathrm{ha}^{-1}\right)
$$

where $\mathrm{C}$ is the organic carbon content at the subsequent genetic horizon (\%), D is the bulk density $\left(\mathrm{g} \cdot \mathrm{cm}^{-3}\right)$, and $\mathrm{m}$ is the thickness of the horizons $(\mathrm{cm})$.

Moreover, a Carbon Distribution Index (CDI) was provided and calculated from the ratio of the carbon accumulated in organic horizons and the amount of organic carbon accumulated in the mineral horizons up to $60 \mathrm{~cm}$ according to the formula:

$$
C D I=\frac{C \text { accumulated in organic horizons }\left[\mathrm{Mg} \cdot \mathrm{ha} \mathrm{a}^{-1}\right]}{\text { C accumulated in mineral horizons to } 60 \mathrm{~cm} \mathrm{depth}\left[\mathrm{Mg} \cdot \mathrm{ha}^{-1}\right]}
$$

\subsection{Statistical Analysis}

Basic descriptive statistics, arithmetic mean and standard deviation were used to estimate the soil properties' variability. Tukey's honest significant difference (HSD) test was used to assess differences between means for carbon accumulation in soils. To evaluate the relationships between stock of soil carbon, other soil properties, forest species, and moisture, the Principal Components Analysis (PCA) method was used. Multiple regression models were developed to describe the relationship between the estimated values of carbon content and other soil properties. The statistical significance of the results was verified at the significance level of alpha $=0.05$. All statistical analyzes were performed with Statistica 12 software (2012).

\section{Results}

The investigated soils are characterized by diversification of properties (Table 2). The values of $\mathrm{pH}$ $\mathrm{H}_{2} \mathrm{O}$ and $\mathrm{pH} \mathrm{KCl}$ were in the range from 3.85 to 7.30 and from 3.04 to 5.78 , respectively. A significantly greater $\mathrm{pH}$ was noted in soils of moist and swampy variants (Table 2). For cation content, a similar dependence was recorded (Table 2). The greater Ah and Aex were observed in soils with water table $80-100 \mathrm{~cm}$ (Table 3). The texture of the investigated soils was dominated by sand (on average $50-72 \%$ in the deeper horizons) and silt (on average $23-42 \%$ in deeper horizons) with addition of clay (on average 5-8\% in the deeper horizon) (Table 3). The sand content (only in the deepest horizons) was significantly greater in $\mathrm{WT}_{80-100}$ variant (Table 3). The silt and clay content (only in the deepest horizons) was higher in soils of moist and swampy variants (Table 3). The analyzed soils clearly differ in their moisture content (Table 4). Statistically significant higher soil moisture content was recorded in the soils of the swampy variant (mean soil moisture of the surface humus horizon was $46.85 \%$ ). The lowest soil moisture content was recorded in the soils of the $\mathrm{WT}_{80-100}$ variant (Table 4). 
Table 2. Chemical properties of study soils.

\begin{tabular}{|c|c|c|c|c|c|c|c|c|c|c|c|c|c|c|c|}
\hline $\begin{array}{l}\text { Moisture } \\
\text { Gradient }\end{array}$ & Horizon & $\mathrm{pH} \mathrm{H}_{2} \mathrm{O}$ & pH KCl & Ah & Aex & Al & $\mathbf{P}$ & $\mathrm{Ca}$ & K & $\mathrm{Mg}$ & $\mathrm{Na}$ & ВС & BS & CECt & CECe \\
\hline \multirow{3}{*}{$\mathrm{WT}_{80-100}$} & $\mathrm{O}$ & $3.85^{b} \pm 0.37$ & $3.04^{b} \pm 0.35$ & $\begin{array}{c}49.68^{\mathrm{a}} \pm \\
18.74\end{array}$ & $\begin{array}{c}10.14^{\mathrm{a}} \pm \\
3.43\end{array}$ & $\begin{array}{c}306.98 \pm \\
243.27\end{array}$ & $\begin{array}{c}58.75^{\mathrm{a}} \pm \\
32.41\end{array}$ & $6.88^{b} \pm 3.30$ & $\begin{array}{c}0.69^{\mathrm{a}} \pm \\
0.24\end{array}$ & $\begin{array}{c}1.21^{\mathrm{a}} \pm \\
0.58\end{array}$ & $0.09^{\mathrm{a}} \pm 0.08$ & $8.89^{b} \pm 3.84$ & $\begin{array}{c}16.49^{\mathrm{b}} \pm \\
8.82\end{array}$ & $\begin{array}{c}58.58^{\mathrm{a}} \pm \\
20.86\end{array}$ & $\begin{array}{l}19.03^{\mathrm{a}} \pm \\
6.07\end{array}$ \\
\hline & $\mathrm{AE}$ & $3.87^{c} \pm 0.16$ & $3.07^{c} \pm 0.18$ & $12.14^{\mathrm{a}} \pm 4.91$ & $\begin{array}{c}4.19^{\mathrm{a}} \pm \\
0.84\end{array}$ & $\begin{array}{c}193.96 \pm \\
79.65\end{array}$ & $8.67^{a} \pm 4.90$ & $0.94^{b} \pm 0.32$ & $\begin{array}{c}0.05^{\mathrm{a}} \pm \\
0.03\end{array}$ & $\begin{array}{c}0.13^{\mathrm{b}} \pm \\
0.09\end{array}$ & $\begin{array}{c}0.02^{\mathrm{b}} \pm \\
0.01\end{array}$ & $1.13^{\mathrm{b}} \pm 0.41$ & $7.15^{b} \pm 4.57$ & $\begin{array}{c}13.26^{\mathrm{a}} \pm \\
6.07\end{array}$ & $5.32^{b} \pm 1.82$ \\
\hline & G & $4.51^{\mathrm{b}} \pm 0.41$ & $3.81^{b} \pm 0.33$ & $3.49^{\mathrm{a}} \pm 1.00$ & $\begin{array}{c}2.21 \mathrm{a} \pm \\
0.63\end{array}$ & $\begin{array}{c}95.36 \pm \\
41.21\end{array}$ & $\begin{array}{c}61.46^{\mathrm{a}} \pm \\
46.18\end{array}$ & $0.36^{\mathrm{b}} \pm 0.19$ & $\begin{array}{c}0.01^{\mathrm{b}} \pm \\
0.01\end{array}$ & $\begin{array}{c}0.03^{\mathrm{b}} \pm \\
0.02\end{array}$ & $\begin{array}{c}0.01^{\mathrm{b}} \pm \\
0.01\end{array}$ & $0.41^{b} \pm 0.37$ & $\begin{array}{c}10.03^{\mathrm{b}} \pm \\
6.19^{-1}\end{array}$ & $3.91^{b} \pm 0.87$ & $2.62^{b} \pm 0.54$ \\
\hline \multirow{3}{*}{ Moist } & A & $4.94^{\mathrm{a}} \pm 0.73$ & $3.98^{\mathrm{a}} \pm 0.69$ & $\begin{array}{c}27.57^{\mathrm{a}, \mathrm{b}} \pm \\
2.10\end{array}$ & $\begin{array}{c}3.04^{\mathrm{b}} \pm \\
1.80\end{array}$ & $69.90 \pm 9.37$ & $\begin{array}{c}19.18^{\mathrm{b}} \pm \\
12.18\end{array}$ & $\begin{array}{c}19.95^{\mathrm{a}} \pm \\
12.86\end{array}$ & $\begin{array}{c}0.36^{\mathrm{b}} \pm \\
0.29\end{array}$ & $1.6^{\mathrm{a}} \pm 1.15$ & $\begin{array}{c}0.09^{\mathrm{a}} \pm \\
0.06\end{array}$ & $\begin{array}{c}22.04^{\mathrm{a}, \mathrm{b}} \pm \\
14.22\end{array}$ & $\begin{array}{c}45.65^{\mathrm{a}} \pm \\
20.06\end{array}$ & $\begin{array}{c}49.61^{\mathrm{a}} \pm \\
31.46\end{array}$ & $\begin{array}{c}25.08^{\mathrm{a}} \pm \\
14.23 \\
\end{array}$ \\
\hline & AG & $5.64^{b} \pm 0.84$ & $4.69^{b} \pm 0.79$ & $\begin{array}{c}7.97 \mathrm{a} a \mathrm{~b} \pm \\
6.17\end{array}$ & $\begin{array}{c}1.27^{\mathrm{b}} \pm \\
0.99\end{array}$ & $\begin{array}{c}35.45 \pm \\
68.05\end{array}$ & $3.85^{b} \pm 3.69$ & $\begin{array}{c}16.07^{\mathrm{a}} \pm \\
14.54\end{array}$ & $\begin{array}{c}0.06^{\mathrm{a}} \pm \\
0.04\end{array}$ & $\begin{array}{c}0.93^{\mathrm{a}} \pm \\
0.87\end{array}$ & $\begin{array}{c}0.06^{\mathrm{a}} \pm \\
0.04\end{array}$ & $\begin{array}{c}17.12^{\mathrm{a}} \pm \\
12.15\end{array}$ & $\begin{array}{c}60.75^{\mathrm{a}} \pm \\
23.38\end{array}$ & $\begin{array}{c}25.10^{\mathrm{a}} \pm \\
19.31\end{array}$ & $\begin{array}{c}18.39^{\mathrm{a}} \pm \\
15.02\end{array}$ \\
\hline & G & $6.85^{a} \pm 0.73$ & $5.26^{\mathrm{a}} \pm 0.58$ & $1.87^{b} \pm 0.65$ & $\begin{array}{c}0.66^{\mathrm{b}} \pm \\
0.17\end{array}$ & $4.14 \pm 5.25$ & $1.27^{b} \pm 0.72$ & $8.30^{a} \pm 5.07$ & $\begin{array}{c}0.07 \mathrm{a} \pm \\
0.05\end{array}$ & $\begin{array}{c}0.60^{\mathrm{a}} \pm \\
0.40\end{array}$ & $\begin{array}{c}0.04 \mathrm{a} \pm \\
0.02\end{array}$ & $9.01^{\mathrm{a}} \pm 5.52$ & $\begin{array}{c}76.92 \mathrm{a} \pm \\
15.04\end{array}$ & $\begin{array}{c}10.88^{\mathrm{a}} \pm \\
5.61\end{array}$ & $9.67^{a} \pm 5.44$ \\
\hline \multirow{3}{*}{ Swampy } & AM & $5.71^{\mathrm{a}} \pm 0.78$ & $4.40^{\mathrm{a}} \pm 1.50$ & $\begin{array}{c}13.01^{\mathrm{b}} \pm \\
11.01\end{array}$ & $\begin{array}{c}1.66^{\mathrm{b}} \pm \\
1.01\end{array}$ & $14.99 \pm 5.84$ & $\begin{array}{c}30.70^{\mathrm{a}, \mathrm{b}} \pm \\
28.43\end{array}$ & $\begin{array}{c}28.73^{\mathrm{a}} \pm \\
15.79\end{array}$ & $\begin{array}{c}0.34^{\mathrm{b}} \pm \\
0.28\end{array}$ & $\begin{array}{c}1.74^{\mathrm{a} \pm} \\
1.12\end{array}$ & $\begin{array}{c}0.10^{\mathrm{a}} \pm \\
0.07\end{array}$ & $\begin{array}{c}30.90^{\mathrm{a}} \pm \\
16.96\end{array}$ & $\begin{array}{c}68.52^{\mathrm{a}} \pm \\
18.79\end{array}$ & $\begin{array}{c}43.92^{\mathrm{a}} \pm \\
25.71\end{array}$ & $\begin{array}{l}32.57^{\mathrm{a}} \pm \\
17.45\end{array}$ \\
\hline & AG & $6.72^{a} \pm 0.61$ & $5.67^{\mathrm{a}} \pm 0.58$ & $3.92^{b} \pm 1.75$ & $\begin{array}{c}0.62^{\mathrm{b}} \pm \\
0.22\end{array}$ & $3.76 \pm 6.59$ & $8.86^{\mathrm{a}} \pm 5.88$ & $\begin{array}{c}21.73^{\mathrm{a}} \pm \\
12.85\end{array}$ & $\begin{array}{c}0.10^{\mathrm{a}} \pm \\
0.08\end{array}$ & $\begin{array}{l}1.09^{\mathrm{a}} \pm \\
0.72\end{array}$ & $\begin{array}{c}0.07^{a} \pm \\
0.05\end{array}$ & $\begin{array}{c}22.999^{\mathrm{a}} \pm \\
13.05\end{array}$ & $\begin{array}{c}82.32^{\mathrm{a}} \pm \\
12.04\end{array}$ & $\begin{array}{c}26.92^{\mathrm{a}} \pm \\
14.75\end{array}$ & $\begin{array}{c}23.62 \mathrm{a} \pm \\
13.45\end{array}$ \\
\hline & G & $7.30^{a} \pm 0.69$ & $5.78^{\mathrm{a}} \pm 0.67$ & $1.75^{\mathrm{b}} \pm 0.91$ & $\begin{array}{c}0.71^{\mathrm{b}} \pm \\
0.29\end{array}$ & $4.11 \pm 1.01$ & $5.09^{\mathrm{a}} \pm 4.47$ & $\begin{array}{c}10.59^{\mathrm{a}} \pm \\
7.06\end{array}$ & $\begin{array}{c}0.12^{\mathrm{a}} \pm \\
0.07\end{array}$ & $\begin{array}{c}0.70^{\mathrm{a}} \pm \\
0.53\end{array}$ & $\begin{array}{c}0.04^{\mathrm{a}} \pm \\
0.02\end{array}$ & $\begin{array}{c}11.40^{\mathrm{a}} \pm \\
7.59\end{array}$ & $\begin{array}{c}83.53^{\mathrm{a}} \pm \\
9.73\end{array}$ & $\begin{array}{c}13.16^{\mathrm{a}} \pm \\
8.22\end{array}$ & $\begin{array}{c}12.11^{\mathrm{a}} \pm \\
7.47\end{array}$ \\
\hline
\end{tabular}

mean \pm standard deviation, small letters (a, b, c) in the upper index of the mean values mean significant differences in soil horizons between the moisture variant; $\mathrm{WT}_{80-100}$, moist and swampy—soil moisture gradient; hydrolytic acidity $(\mathrm{Ah})\left(\mathrm{cmol}(+) \cdot \mathrm{kg}^{-1}\right)$; hydrolytic acidity $(\mathrm{Ah})\left(\mathrm{cmol}(+) \cdot \mathrm{kg}^{-1}\right) ; \mathrm{Ca}, \mathrm{K}, \mathrm{Mg}$ and $\mathrm{Na}\left(\mathrm{cmol}(+) \cdot \mathrm{kg}^{-1}\right) ; \mathrm{Al}, \mathrm{P}\left(\mathrm{mg} \cdot \mathrm{kg}^{-1}\right) ;$ total cation exchange capacity $(\mathrm{CECt})\left(\mathrm{cmol}(+) \cdot \mathrm{kg}^{-1}\right)$; effective cation exchange capacity (CECe) $\left(\mathrm{cmol}(+) \cdot \mathrm{kg}^{-1}\right)$; base cations $(\mathrm{BC})\left(\mathrm{cmol}(+) \cdot \mathrm{kg}^{-1}\right)$; base saturation (BS) $(\%)$. 
Table 3. The particle size $[\%]$ and bulk density $\left[\mathrm{g} \cdot \mathrm{cm}^{-3}\right]$ of study soils.

\begin{tabular}{|c|c|c|c|c|c|c|c|c|c|c|c|c|}
\hline \multirow{2}{*}{$\begin{array}{l}\text { Moisture } \\
\text { Gradient }\end{array}$} & \multirow{2}{*}{ Horizon } & \multicolumn{5}{|c|}{ Sand Fraction } & \multicolumn{2}{|c|}{ Silt Fraction } & \multirow{2}{*}{ Clay } & \multicolumn{2}{|c|}{ Sum of Fraction } & \multirow{2}{*}{ BD } \\
\hline & & VCS & CS & MS & FS & VFS & Csi & Fsi & & Sand & Silt & \\
\hline \multirow{3}{*}{$\mathrm{WT}_{80-100}$} & $\mathrm{O}$ & n.d. & n.d. & n.d. & n.d. & n.d. & n.d. & n.d. & n.d. & n.d. & n.d. & $0.41^{\mathrm{b}} \pm 0.21$ \\
\hline & $\mathrm{AE}$ & $0.4^{\mathrm{a}} \pm 0.3$ & $15.5^{\mathrm{a}} \pm 8.8$ & $30.5^{\mathrm{a}} \pm 2.6$ & $15.0^{\mathrm{a}} \pm 4.0$ & $9.2^{\mathrm{a}} \pm 2.4$ & $10.9^{\mathrm{a}} \pm 3.7$ & $14.6^{\mathrm{a}} \pm 4.5$ & $3.8^{a} \pm 1.3$ & $70.7^{\mathrm{a}} \pm 8.4$ & $25.5^{\mathrm{a}} \pm 7.3$ & $1.14^{\mathrm{a}} \pm 0.11$ \\
\hline & G & $1.3^{\mathrm{a}} \pm 0.7$ & $24.8^{b} \pm 10.5$ & $31.5^{\mathrm{a}} \pm 3.2$ & $10.6^{\mathrm{a}} \pm 3.8$ & $3.5^{\mathrm{a}} \pm 1.3$ & $7.4^{\mathrm{b}} \pm 3.0$ & $15.9^{b} \pm 6.2$ & $4.9^{b} \pm 1.8$ & $71.6^{\mathrm{a}} \pm 9.1$ & $23.4^{b} \pm 7.6$ & $1.34^{\mathrm{a}} \pm 0.01$ \\
\hline \multirow{3}{*}{ Moist } & A & $1.1^{\mathrm{a}} \pm 1.0$ & $10.9^{\mathrm{a}} \pm 8.4$ & $19.7^{\mathrm{a}} \pm 11.4$ & $18.9^{\mathrm{a}} \pm 8.1$ & $11.26^{a} \pm 5.3$ & $11.6^{\mathrm{a}} \pm 7.2$ & $11.6^{\mathrm{a}} \pm 9.1$ & $2.7^{\mathrm{a}} \pm 2.0$ & $62.0^{\mathrm{a}} \pm 30.1$ & $23.3^{\mathrm{a}} \pm 15.7$ & $0.83^{a} \pm 0.25$ \\
\hline & AG & $0.5^{\mathrm{a}} \pm 0.2$ & $8.9^{a} \pm 6.1$ & $20.1^{b} \pm 10.6$ & $17.5^{\mathrm{a}} \pm 3.5$ & $10.8^{\mathrm{a}} \pm 3.7$ & $16.8^{\mathrm{a}} \pm 6.8$ & $21.2^{\mathrm{a}} \pm 9.5$ & $4.3^{\mathrm{a}} \pm 2.1$ & $57.0^{\mathrm{a}} \pm 17.5$ & $37.9^{\mathrm{a}} \pm 15.9$ & $1.21^{\mathrm{a}} \pm 0.08$ \\
\hline & G & $0.3^{\mathrm{a}} \pm 0.1$ & $7.8^{\mathrm{a}} \pm 5.7$ & $23.7^{a} \pm 11.5$ & $14.4^{\mathrm{a}} \pm 3.2$ & $4.9^{\mathrm{a}} \pm 2.0$ & $14.0^{\mathrm{a}} \pm 6.5$ & $27.1^{\mathrm{a}} \pm 10.9$ & $7.7^{a} \pm 2.9$ & $51.1^{\mathrm{b}} \pm 18.8$ & $41.1^{\mathrm{a}} \pm 16.3$ & $1.35^{\mathrm{a}} \pm 0.02$ \\
\hline \multirow{3}{*}{ Swampy } & $\mathrm{AM}$ & $1.3^{a} \pm 0.8$ & $13.0^{\mathrm{a}} \pm 3.0$ & $19.3^{a} \pm 12.9$ & $16.3^{\mathrm{a}} \pm 10.8$ & $7.1^{\mathrm{a}} \pm 6.0$ & $6.9^{a} \pm 5.1$ & $6.9^{a} \pm 6.3$ & $1.9^{\mathrm{a}} \pm 1.0$ & $64.7^{a} \pm 33.3$ & $15.1^{\mathrm{a}} \pm 11.0$ & $0.69^{\mathrm{a}, \mathrm{b}} \pm 0.31$ \\
\hline & AG & n.d. & $9.1^{\mathrm{a}} \pm 7.1$ & $20.3^{\mathrm{a}, \mathrm{b}} \pm 12.4$ & $18.2^{\mathrm{a}} \pm 5.5$ & $9.5^{\mathrm{a}} \pm 3.9$ & $14.8^{\mathrm{a}} \pm 7.3$ & $21.9^{\mathrm{a}} \pm 12.9^{2}$ & $5.3^{\mathrm{a}} \pm 2.9$ & $57.8^{\mathrm{a}} \pm 22.2$ & $36.8^{\mathrm{a}} \pm 19.4$ & $1.07^{\mathrm{a}} \pm 0.19$ \\
\hline & G & n.d. & $5.7^{a} \pm 2.6$ & $22.7^{a} \pm 12.6$ & $15.4^{\mathrm{a}} \pm 6.8$ & $5.6^{\mathrm{a}} \pm 3.4$ & $12.9^{b} \pm 8.6$ & $28.7^{a} \pm 11.1$ & $7.8^{a} \pm 3.5$ & $49.5^{b} \pm 19.8$ & $41.6^{\mathrm{a}} \pm 18.7$ & $1.31^{\mathrm{a}} \pm 0.08$ \\
\hline
\end{tabular}

n.d. no determine, mean \pm standard deviation, small letters $(a, b, c)$ in the upper index of the mean values mean significant differences in soil horizons between the moisture variant,

$\mathrm{WT}_{80-100}$, moist and swampy—soil moisture gradient; VCS—very coarse sand, CS—coarse sand, MS—-medium sand, FS—fine sand, VFS—very fine sand, CSi—coarse silt, FSi-fine silt; BD-bulk density.

Table 4. Humus substances, organic carbon and nitrogen content, moisture of study soils.

\begin{tabular}{|c|c|c|c|c|c|c|c|c|c|c|c|c|}
\hline $\begin{array}{l}\text { Moisture } \\
\text { Gradient }\end{array}$ & Horizon & $\mathrm{C}$ & $\mathbf{N}$ & $\mathrm{C} / \mathrm{N}$ & HA & FA & $\mathrm{Hm}$ & HA/HF & $\mathrm{HA}+\mathrm{HF} / \mathrm{Hm}$ & SOCs & CDI & Moisture \\
\hline \multirow{3}{*}{$\mathrm{WT}_{80-100}$} & $\mathrm{O}$ & $241.87^{\mathrm{a}} \pm 74.21$ & $12.45^{\mathrm{a}} \pm 5.28$ & $19.6^{b} \pm 2.8$ & $11.9^{\mathrm{a}} \pm 6.78$ & $4.43^{\mathrm{a}} \pm 2.28$ & n.d. & $2.74^{a} \pm 1.12$ & n.d. & \multirow{3}{*}{$14.09^{\mathrm{a}, \mathrm{b}} \pm 2.98$} & \multirow{3}{*}{0.837} & \multirow{3}{*}{$16.43^{c} \pm 3.42$} \\
\hline & $\mathrm{AE}$ & $35.07^{a} \pm 19.41$ & $1.75^{\mathrm{a}} \pm 1.15$ & $21.1^{\mathrm{b}} \pm 3.1$ & $3.08^{\mathrm{a}} \pm 1.44$ & $0.63^{a} \pm 0.61$ & $0.47^{\mathrm{a}} \pm 1.03$ & $4.70^{a} \pm 1.38$ & $8.00^{\mathrm{a}} \pm 5.03$ & & & \\
\hline & G & $4.87^{\mathrm{a}, \mathrm{b}} \pm 2.01$ & $0.24^{\mathrm{a}} \pm 0.07$ & $20.3^{b} \pm 2.5$ & $0.81^{\mathrm{a}} \pm 0.44$ & $0.82^{a} \pm 0.45$ & $0.3^{a} \pm 0.03$ & $1.05^{\mathrm{a}} \pm 1.10$ & $54.30^{\mathrm{a}} \pm 22.90$ & & & \\
\hline \multirow{3}{*}{ Moist } & A & $101.10^{b} \pm 49.29$ & $6.80^{\mathrm{a}} \pm 3.79$ & $14.2^{\mathrm{a}} \pm 1.7$ & $3.15^{b} \pm 3.04$ & $3.41^{\mathrm{a}} \pm 3.55$ & $9.79^{a} \pm 10.41$ & $1.80^{\mathrm{a}} \pm 2.31$ & $0.68^{\mathrm{b}} \pm 0.84$ & \multirow{3}{*}{$12.61^{\mathrm{b}} \pm 3.71$} & \multirow{3}{*}{0.299} & \multirow{3}{*}{$30.08^{b} \pm 4.8 \mathrm{e}$} \\
\hline & AG & $24.29^{\mathrm{a}} \pm 13.80$ & $1.84^{\mathrm{a}} \pm 1.04$ & $13.2^{\mathrm{a}} \pm 1.2$ & $1.37^{b} \pm 0.73$ & $1.69^{a} \pm 2.10$ & $1.89^{\mathrm{a}} \pm 2.53$ & $2.25^{b} \pm 1.96$ & $1.63^{\mathrm{b}} \pm 1.25$ & & & \\
\hline & G & $3.95^{\mathrm{b}} \pm 2.30$ & $0.33^{\mathrm{a}} \pm 0.18$ & $11.8^{\mathrm{a}} \pm 1.1$ & $0.99^{\mathrm{a}} \pm 1.12$ & $0.72^{a} \pm 0.94$ & $0.25^{a} \pm 0.26$ & $3.31^{\mathrm{a}} \pm 1.18$ & $11.47^{a} \pm 8.98$ & & & \\
\hline \multirow{3}{*}{ Swampy } & AM & $135.96^{\mathrm{a}, \mathrm{b}} \pm 107.11$ & $10.09^{a} \pm 7.02$ & $12.8^{\mathrm{a}} \pm 1.6$ & $4.67^{\mathrm{a}, \mathrm{b}} \pm 3.55$ & $2.91^{\mathrm{a}} \pm 3.72$ & $5.51^{\mathrm{a}} \pm 7.63$ & $3.84^{\mathrm{a}} \pm 2.41$ & $1.40^{\mathrm{a}, \mathrm{b}} \pm 0.68$ & \multirow{3}{*}{$19.02^{\mathrm{a}} \pm 1.82$} & \multirow{3}{*}{0.329} & \multirow{3}{*}{$46.85^{a} \pm 3.28$} \\
\hline & AG & $44.13^{\mathrm{a}} \pm 34.29$ & $3.31^{\mathrm{a}} \pm 2.39$ & $13.1^{\mathrm{a}} \pm 1.2$ & $2.26^{\mathrm{a}, \mathrm{b}} \pm 1.56$ & $1.34^{\mathrm{a}} \pm 1.65$ & $1.08^{\mathrm{a}} \pm 1.47$ & $2.51^{\mathrm{b}} \pm 1.06$ & $3.40^{\mathrm{b}} \pm 2.20$ & & & \\
\hline & G & $8.51^{\mathrm{a}} \pm 4.31$ & $0.64^{\mathrm{a}} \pm 0.51$ & $12.8^{\mathrm{a}} \pm 1.6$ & $0.79^{a} \pm 0.72$ & $0.99^{\mathrm{a}} \pm 0.90$ & $0.31^{\mathrm{a}} \pm 0.28$ & $2.98^{\mathrm{a}} \pm 1.47$ & $16.18^{a} \pm 12.45$ & & & \\
\hline
\end{tabular}

mean \pm standard deviation, small letters $(\mathrm{a}, \mathrm{b}, \mathrm{c})$ in the upper index of the mean values mean significant differences in soil horizons between the moisture variant; $\mathrm{WT}$ ( and swampy—-soil moisture gradient; $\mathrm{C}$ and $\mathrm{N}\left(\mathrm{g} \cdot \mathrm{kg}^{-1}\right)$; HA carbon of humic acids (\% C), FA carbon of fulvic acids (\% C), Hm carbon of humin (\% C); SOCs soil organic carbon stock $\left(\mathrm{Mg} \cdot \mathrm{ha}^{-1}\right)$; CDI carbon distribution index; moisture (\%); n.d.-no determine. 
The differences in the carbon content of the examined soils were clearly noted. A statistically higher carbon content in the surface horizons was noted in the soils of the $\mathrm{WT}_{80-100}$ variant. The average content of carbon in the surface horizon of this variant was $241.87 \mathrm{~g} \cdot \mathrm{kg}^{-1}$, and was $101.10 \mathrm{~g} \cdot \mathrm{kg}^{-1}$ and $135.96 \mathrm{~g} \cdot \mathrm{kg}^{-1}$ in the soils of the moist and swampy variants, respectively (Table 4). The highest carbon content in the lower lying horizons was recorded for soils of the swampy variant. The mean carbon content at these horizons was $44.13 \mathrm{~g} \cdot \mathrm{kg}^{-1}$ and $8.51 \mathrm{~g} \cdot \mathrm{kg}^{-1}$, respectively. In the case of $\mathrm{WT}_{80-100}$ and moist variant of soils in the deeper horizons, the carbon content was $35.07-4.87 \mathrm{~g} \cdot \mathrm{kg}^{-1}$ and 24.29-3.95 g. $\mathrm{kg}^{-1}$, respectively. Similar dependencies concerned nitrogen content (Table 4). The lowest $\mathrm{C} / \mathrm{N}$, which expresses the degree of organic matter distribution, was recorded in the soils of the swampy and moist variants (the $\mathrm{C} / \mathrm{N}$ ratio ranged from 11.8 to 14.2 ). The highest $\mathrm{C} / \mathrm{N}$ ratio, from 19.6 to 20.3 depending on the horizon, was recorded in the $\mathrm{WT}_{80-100}$ variant of soils (Table 4). Soil organic matter (SOCs) concentration in the $0-60 \mathrm{~cm}$ soil horizon differed significantly among moisture gradients and followed the order: swampy $>\mathrm{WT}_{80-100}>$ moist variants. The average SOCs up to a depth of $60 \mathrm{~cm}$ in the soils of moist variant is lower and is estimated at $12.61 \mathrm{Mg} \cdot \mathrm{ha}^{-1}$. The higher accumulation was found in the soils of $\mathrm{WT}_{80-100}$ variant, where the average SOCs was $14.09 \mathrm{Mg} \cdot \mathrm{ha}^{-1}$. A significantly greater accumulation was noted in the soils of swampy variant $\left(19.02 \mathrm{Mg} \cdot \mathrm{ha}^{-1}\right)$. The ratio between carbon accumulation in the organic horizon and carbon accumulation in the mineral horizons, up to $60 \mathrm{~cm}$ of soils (CDI) of estimated variants, is notably diverse (average $0.299-0.837$ ). The lowest values of CDI are found in the soils of moist variant in which the CDI indicator is 0.299 . The soils of swampy variant are characterized by higher values of the CDI index- 0.329 , and the CDI index of soils of $\mathrm{WT}_{80-100}$ variant approached 1.0 and is 0.837 (Table 4).

Concentrations of $\mathrm{C}$ in fulvic acid (FA), humic acid (HA) and humin fractions in the different soil horizons of different moisture variants are presented in Table 4. Mean values of HA, FA and humin varied from $11.9 \%$ to $0.79 \%, 4.43 \%$ to $0.63 \%$, and $9.79 \%$ to $0.25 \%$, respectively. In the fractions of SOM, humic acids dominate over fulvic acids and humins. Soils of the $\mathrm{WT}_{80-100}$ variant are characterized by the highest content of humic and fulvic acid (Table 4). Significant differences between the soils in terms of humic acid were observed in two surface horizons (swampy variant $=\mathrm{WT}_{80-100}$ variant $>$ moist variant $=$ swampy variant). No significant differences between soil variant in terms of fulvic acid and humin were noted (Table 4). All SOM fractions decreased significantly with depth for all variants of soil. The HA/FA ratio ranged from 4.70 to 1.05. The lowest HA/FA ratio regardless of depth was in the soil of $\mathrm{WT}_{80-100}$ variant, and the highest in the soil of moist and swampy variant. The relationship of the sum of $\mathrm{C}$ humic and fulvic acids to $\mathrm{C}$ humin $(\mathrm{HA}+\mathrm{FA} / \mathrm{Hm})$ assumes the values of $54.3-0.68$ (Table 4). In the soil of $\mathrm{WT}_{80-100}$ variant, the highest $\mathrm{HA} / \mathrm{FA}$ ratio and $\mathrm{HA}+\mathrm{FA} / \mathrm{Hm}$, as compared to moist and swampy variant, was recorded and was statistically significant (Table 4).

Multiple regression models explained $89 \%$ of the variance in the $C$ content (Table 5 ). $C$ content was largely dependent on hydrolytic acidity and moisture. The relationships between carbon content, hydrolytic acidity and moisture are shown in Figure 2. The increase in C accumulation in soils correlated with high moisture and high acidity.

A projection of the variables on the factor-plane clearly demonstrated a correlation between the variables and soil moisture (supplementary variables). Two main factors had a significant total impact $(60.62 \%)$ on the variance of the variables in soil. Factor 1 explained $35.76 \%$ of the variance of the examined properties and Factor 2 explained $24.86 \%$ of the variance (Figure 3). The soil of moist and swampy variants had a higher $\mathrm{pH}$. In contrast, the soils of $\mathrm{WT}_{80-100}$ variant were characterized by a high $\mathrm{C} / \mathrm{N}$ ratio. The soils of $\mathrm{WT}_{80-100}$ variant contained more humic acids. The fulvic acids and humins were connected with nitrogen content. 
Table 5. Multiple regression analysis for carbon content based on soil characteristic. $R^{2}$ describes the percentage of explained variance, $\beta$ is the regression coefficient for the given equation parameter and $p$ is the significance level for the equation parameter.

\begin{tabular}{ccccc}
\hline & $\boldsymbol{R}^{\mathbf{2}}$ & Equation Parameter & $\boldsymbol{\beta}$ & $\boldsymbol{p}$ \\
\hline \multirow{2}{*}{$\mathrm{C}$} & \multirow{2}{*}{$89 \%$} & Ah & 5.257 & 0.000001 \\
& & Moisture & 1.897 & 0.000001 \\
\hline
\end{tabular}

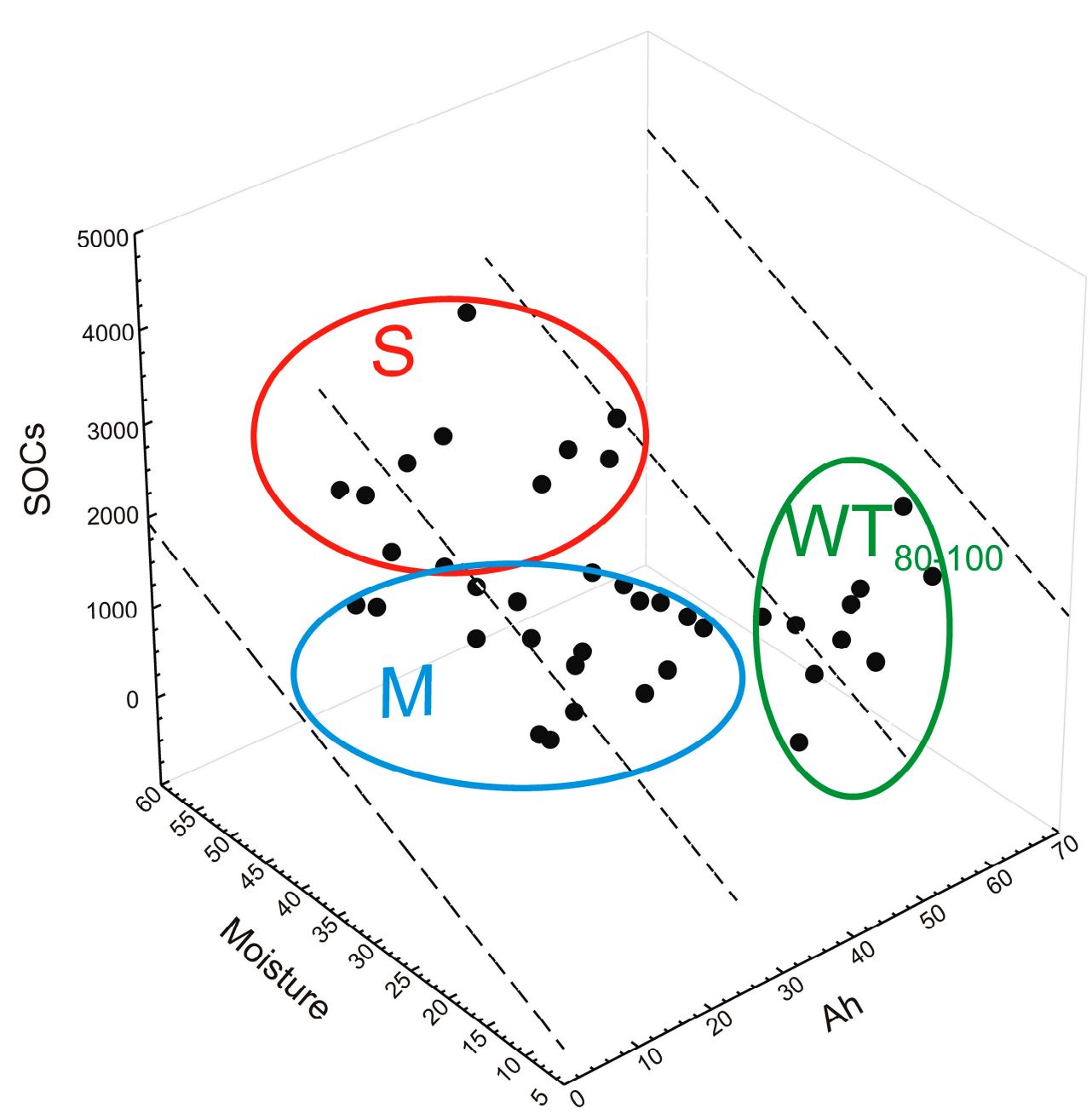

Figure 2. Three-dimensional plot of the relationship between soil organic carbon stock (SOCs), moisture and hydrolytic acidity (Ah) of soils (circles with $\mathrm{WT}_{80-100}$ - group of plots with water table $80-100 \mathrm{~cm}$, circles with $\mathrm{M}$-group of moisture plots, circles with S-group of swampy plots); regression plane marked by black lines. 


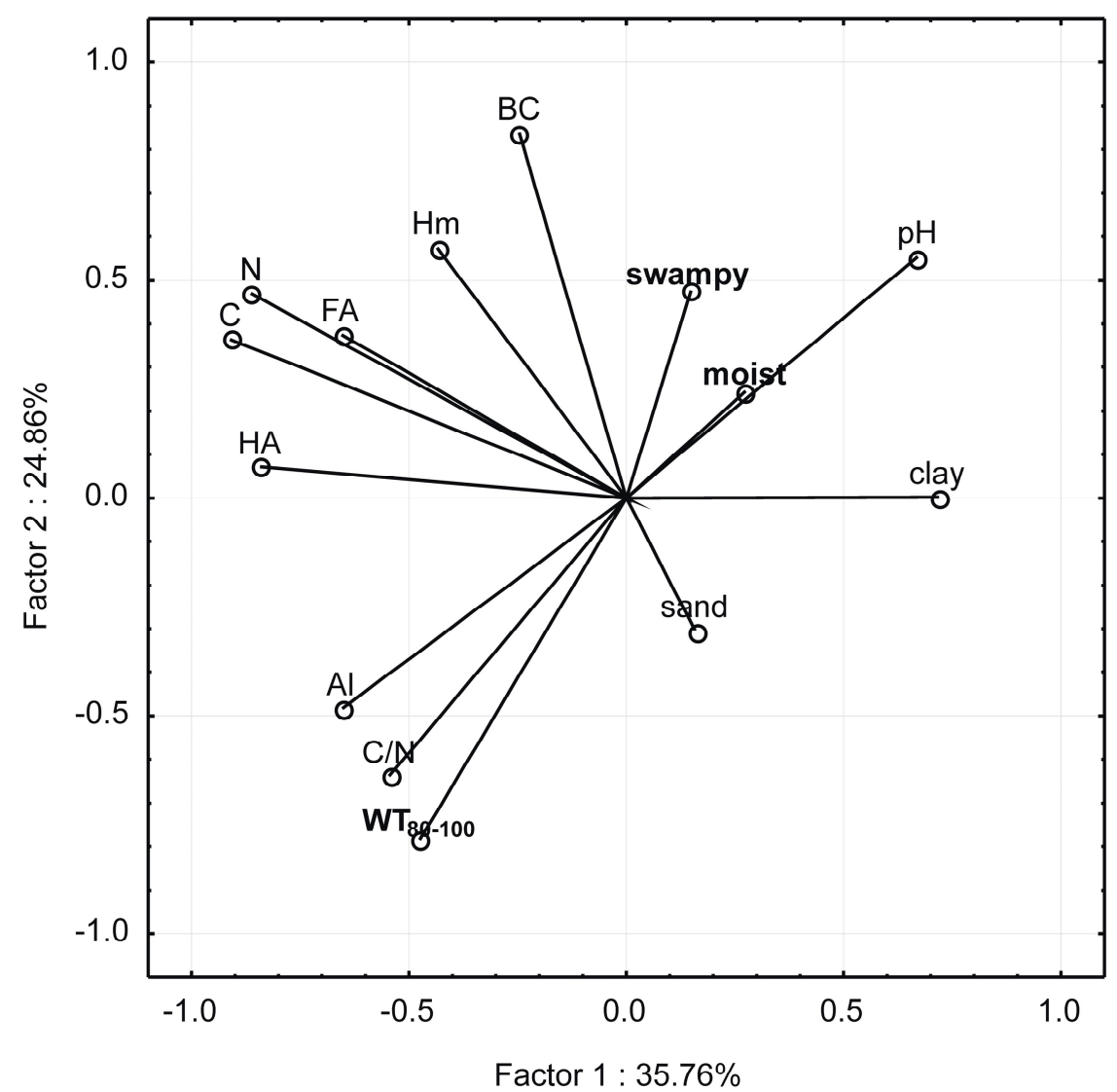

Figure 3. Diagram of Principal Components Analysis (PCA) with projection of variables on a plane of the first and second factor in the surface horizon $\left(\mathrm{WT}_{80-100}\right.$, moist and swampy-soil moisture gradient; HA-humic acids, FA—fulvic acids, Hm-humins).

\section{Discussion}

Results present in this study demonstrate that soil moisture is a factor that most strongly influences the carbon accumulation in forest soils. Compared to the soil with water table from 80 to $100 \mathrm{~cm}$ and moist soils, those with the highest moisture content (swampy variant) are characterized by the higher $\mathrm{C}$ accumulation. Soil organic matter (SOCs) concentration differed significantly among moisture gradient and followed the order: swampy $>\mathrm{WT}_{80-100}>$ moist variants. The greater $\mathrm{SOM}$ accumulation in the soils is affected not only by the high moisture, but also periodic deficiency of water. Our results show the high SOC stock on the sites with low moisture ( $\mathrm{WT}_{80-100}$ variant) and in swampy variant, and that site condition promoted lower rate decomposition. The high SOC stock in the soils of $\mathrm{WT}_{80-100}$ variant is affected by greater acidity (surface horizons are not enriched due to low groundwater level). In the soils of swampy variant, there is an effect of anaerobic processes: the soils better aerated in the moist variants are characterized by faster and more efficient decomposition of SOM. Jandl et al. [26] and Stendahl et al. [27] related the accumulation of SOC with site conditions. A large stock of carbon can be associated with the accumulation of organic matter in acidic environments. A similar situation was noted for the soils of the $\mathrm{WT}_{80-100}$ variant where surface horizons were under coniferous species influence. Tree species affect $\mathrm{pH}$, and the specific composition of organic matter may lead to the modification of soil acidity and nutrient pools [28-30]. $\mathrm{pH}$ is an important variable that influences SOM decomposition dynamics [31]. Our results confirmed the importance of moisture and acidity of soils on soil organic matter accumulation. In our study, $89 \%$ of the variance in the C content was explained by the hydrolytic acidity and moisture of the soils. Li et al.'s [32] results indicated that moisture level had an effect on carbon accumulation. At the same time, the increase of soil's acidity results in deterioration of the conditions of decomposition of dead organic matter, slowing down 
their humification rate and microbial activity. On the other hand, the increase in organic matter accumulation results in an increase in sorption capacity, which, under acidic forest soil condition and lower level of groundwater, includes aluminum and hydrogen ions. The increase in soil moisture was favorable for the accumulation of water soluble carbon. In our study, soils in the swampy variant are characterized by the highest accumulation of $C$, which can be combined with the accumulation of organic debris in the peat-forming process. These studies confirm earlier findings, that although the peat covers only $3 \%$ of the Earth's surface, it constitutes an important reservoir of carbon $[33,34]$. Under anaerobic conditions, 2-6 times less $\mathrm{CO}_{2}$ was produced than under aerobic conditions [35]. Furthermore, the presence of water in the soil profile facilitates dissolution of minerals, and during the capillary transport, also their transportation to higher horizons where they can form stable conjunctions to the humic substances. In addition, higher soil moisture results in higher biomass production of trees and plant cover of lower layers of the stand, which results in increased accumulation of organic material into the forest floor and soil.

In our study, CDI was used to evaluate the distribution of carbon accumulated in the soil profile and as a measure of quality of the accumulated organic matter. The advantage of accumulation of carbon in the mineral horizons over accumulation in the organic horizons indicates a greater efficiency of the processes of organic matter decomposition. In numerous studies [36], the greater resistance of $\mathrm{SOM}$, in combination with soil minerals, is observed. In comparison to the accumulation occurring in the organic horizons, the predominance of carbon accumulation in the mineral horizons can be observed in soils of moist and swampy variants. The soils of moist variants were occupied by a very diverse species composition; the presence of admixture species-mainly hornbeam, alder and maple-provides abundant input of easily decaying organic matter to the soil. The positive impact of hornbeam on the accumulation of SOM was also described by other authors [31,37]. Stand mixing improves soil properties, especially the quality of SOM and biochemical properties [38]. The dominance of coniferous species in the forest ( $\mathrm{WT}_{80-100}$ variant) changes the CDI index. In coniferous forests with fir, the $C$ accumulation in the mineral horizons is reduced in relation to the total accumulation in the whole soil profile. The reason for the higher accumulation of $C$ at the organic horizons is undoubtedly the slow decomposition of organic debris. Litter decomposition is particularly influenced by decomposer organisms [39]. The conifer litter decomposes slower than that from hardwood species [40]. The soils of coniferous forests are characterized by low $\mathrm{pH}$, and the decomposition of litter is mainly conducted by fungal organisms. Fungi dominate in acidic soils, whilst bacteria in soils are characterized by a neutral or alkaline $\mathrm{pH}$ [41].

Forest soils in the studied moisture content gradient differ in the accumulation of carbon and the fractional composition of the soil organic matter. In the studied soils, a large proportion of humic acids were found, which prevailed in the moist variants. This is in line with Tavares and Nahas' study [42]. More humic acids than fulvic acids indicate the potentially low mobility of carbon accumulated in the surface soil horizons [21]. In our studies, the ratio of humic to fulvic acids (HA/FA) changes as moisture content of soils increases, suggesting an increase in carbon mobility in these soils. More humic acids than fulvic acids indicate high humification rates. The differences in soil moisture contents influences the amounts and chemical composition of the soil humic fraction [32]. The results implied that a high moisture level was unfavorable for the accumulation of humic fraction in the soil. In our experiment, we used the ratio between the sum of humic and fulvic acids and humin ((HA+FA)/humin) as an indicator of loss of SOM through the soil profile. The highest ratio ((HA+FA)/humin) was determined in the soils with the lowest moisture. As the soil moisture content increased, the ratio decreased. The lower ratio ((HA+FA)/humin) indicates the sufficient humin level in the soils with the highest moisture content.

\section{Conclusions}

In the soils under study, in the temperate zone, moisture is an important factor in the accumulation of organic carbon in the soil. The highest accumulation of carbon was observed in soils of swampy 
variant, while the lowest in the soils of moist variant. In the soil of swampy variant, the anaerobic conditions affect the organic matter decomposition leading to slower processes. The increased accumulation of $C$ in soils of $\mathrm{WT}_{80-100}$ variant is caused by other factors. Large accumulation of $\mathrm{C}$ in the soils of the $\mathrm{WT}_{80-100}$ variant results from the thick organic horizons that are characterized by lower organic matter decomposition and higher acidity. In the $\mathrm{WT}_{80-100}$ variant, the higher acidity is simultaneously an effect of the stand species composition and lack of water neutralization. The proportion of carbon accumulation in the organic horizons to the total accumulation in the mineral horizons expresses the distribution of carbon accumulated in the soil profile, and is a measure of quality of the organic matter accumulated. The advantage of accumulation at the mineral horizons over accumulation at the organic horizons confirms a greater efficiency of decomposition processes of organic matter, which, thanks to more advanced transformations and connecting with mineral soil, can be considered stable. Studies have confirmed the importance of moisture content in the formation of the fractional organic matter. The increase in soil moisture content changes the ratio of humic to fulvic acids (HA/FA), which may suggest an increase in carbon mobility in soils.

Acknowledgments: The project was financed by the National Science Centre, Poland: decision no. DEC-2016/21/D/NZ9/01333.

Author Contributions: E.B. conceived and designed the experiments, performed the experiments, wrote the paper; J.L. analyzed the data.

Conflicts of Interest: The authors declare no conflict of interest.

\section{References}

1. Dorrepaal, E.; Sylvia, T.; van Logtestijn, R.S.P.; Swart, E.; Van de Weg, M.J.; Callaghan, T.V.; Aerts, R. Carbon respiration from subsurface peat accelerated by climate warming in the subarctic. Nature 2009, 460, 616-619. [CrossRef]

2. Fornara, D.A.; Steinbeiss, S.; McNamara, N.P.; Gleixner, G.; Oakley, S.; Poulton, P.R. Increases in soil organic carbon sequestration can reduce the global warming potential of long-term liming to permanent grassland. Glob. Chang. Biol. 2011, 17, 2762. [CrossRef]

3. Farquhar, G.D.; Fasham, M.J.R.; Goulden, M.L.; Heimann, M.; Jaramillo, V.J.; Kheshgi, H.S.; Le Quere, C.; Scholes, R.J.; Wallace, D.W.R. Climate change. The Scientific Basis IPCC. Chapter 3. In The Carbon Cycle and Atmospheric Carbon Dioxide; Cambridge University Press: Cambridge, UK, 2001; pp. 183-237.

4. Stergiadi, M.; Van der Peck, M.; de Nijs, T.C.M.; Bierkens, M.F.P. Effect of climate change and land management on soil organic carbon dynamics and carbon leaching in northwestern Europe. Biogeosciences 2016, 13, 1519-1536. [CrossRef]

5. Martin, M.P.; Wattenbach, M.; Smith, P.; Meersmans, J.; Jolivet, C.; Boulonne, L.; Arrouays, D. Spatial distribution of soil organic carbon stock in France. Biogeosciences 2011, 8, 1053-1065. [CrossRef]

6. Parajuli, P.B.; Duffy, S. Evaluation of Soil Organic Carbon and Soil Moisture Content from Agricultural Fileds in Mississipi. Open J. Soil Sci. 2013, 3, 81-90. [CrossRef]

7. Hobley, E.U.; Wilson, B. The depth distribution of organic carbon in the soils of eastern Australia. Ecosphere 2016, 7, e01214. [CrossRef]

8. Degórski, M. Influence of forest management into the carbon storage. Monit. Environ. 2005, 6, 75-83.

9. Zwydak, M.; Brożek, S.; Lasota, J.; Małek, S. Reserve of Organic Carbon in Forest Soils of Lowlands in Poland. Pol. J. Environ. Stud. 2008, 17, 632-637.

10. Ostrowska, A.; Porębska, G.; Kanafa, M. Carbon accumulation and Distribution in Profiles of Forest Soils. Pol. J. Environ. Stud. 2010, 19, 1307-1315.

11. Stavi, I. Biochar use in forestry and tree-based agro-ecosystems for increasing climate change mitigation and adaptation. Int. J. Sustain. Dev. World Ecol. 2013, 20, 166-181. [CrossRef]

12. Lasota, J.; Błońska, E. Forest Site Science in the Polish Lowlands and Highlands; Scientific Papers; University of Agriculture in Krakow: Kraków, Poland, 2013.

13. Hassink, J. The capacity of soils to preserze organic $\mathrm{C}$ and $\mathrm{N}$ by their association with Clay and silt particles. Plant Soil 1997, 191, 77-87. [CrossRef] 
14. Six, J.; Paul, E.; Paustian, K. Stabilization Maechanisms of Soil Organic Matter: Implications for C-Saturation of Soils. Plant Soil 2002, 241, 155-176. [CrossRef]

15. Wosten, J.H.; Lilly, A.; Nemes, A.; Le Bas, C. Development and use of a database of hydraulic properties of European soils. Geoderma 1999, 90, 169-185. [CrossRef]

16. Bauer, J.; Herbst, M.; Huisman, J.A.; Weihermuller, L.; Vereecken, H. Sensitivity of simulated soil heterotrophic respiration to temperature and moisture reduction functions. Geoderma 2008, 145, 17-27. [CrossRef]

17. Meersmans, J.; De Ridder, F.; Canters, F.; De Baets, S.; Van Molle, M. A multiple regression approach to assess the spatial distribution of Soil Organic Carbon (SOC) at the regional scale (Flanders, Belgium). Geoderma 2008, 143, 1-13. [CrossRef]

18. Frouz, J.; Kalčík, J. Accumulation of soil organic carbon in relation to other soil characteristic during spontaneous succession in non-reclaimed colliery spoil heaps after brown coal mining near Sokolov (the Czech Republic). Ekológia 2006, 25, 388-397.

19. Haynes, R.J. Labile organic matter fractions as central components of the quality of agricultural soils: An overview. Adv. Agron. 2005, 85, 221-268.

20. Dębska, B.; Długosz, J.; Piotrowska-Długosz, A.; Banach-Szott, M. The impact of a bio-fertilizer on the soil organic matter status and carbon sequestration—Results from a field-scale study. J. Soils Sediments 2016, 16, 2335-2343. [CrossRef]

21. Guimarães, D.V.; Gonzaga, M.I.S.; da Silva, T.O.; da Silva, T.L.; Dias, N.D.; Matias, M.I.S. Soil organic matter pools and carbon fractions in soil under different land uses. Soil Till. Res. 2013, 126, 177-182. [CrossRef]

22. Błońska, E.; Lasota, J.; Piaszczyk, P.; Wiecheć, M.; Klamerus-Iwan, A. The effect of landslide on soil organic carbon stock and biochemical properties of soil. J. Soil Sendiments 2017. [CrossRef]

23. World Reference Base (WRB). World Reference Base for Soil Resource; FAO: Rome, Italy, 2014.

24. Dziadowiec, H.; Gonet, S. Przewodnik metodyczny do badań materii organicznej gleb. Prace Komisji Naukowych PTG 1999, 20, 42-43.

25. Grossman, R.B.; Reinsch, T.G. Bulk density and linear extensibility. In Methods of Soil Analysis, Part 4; Dane, J.H., Topp, G.C., Eds.; Soil Science Society of America: Madison, WI, USA, 2002; pp. 201-225.

26. Jandl, R.; Lindner, M.; Vesterdal, L.; Bauwens, B.; Baritz, R.; Hagedorn, F.; Johnson, D.W.; Minkkinen, K.; Byrne, K.A. How strongly can forest management influence soil carbon sequestration? Geoderma 2007, 137, 253-268. [CrossRef]

27. Stendhal, J.; Johansson, M.B.; Eriksson, E.; Langvall, O. Soil organic carbon in Swedish spruce and pine forests-differences in stock levels and regional patterns. Silva Fennica 2010, 33, 5-21. [CrossRef]

28. Prescott, P.C.; Grayston, S.J. Tree species influence on microbial communities in litter and soil: Current knowledge and research needs. For. Ecol. Manag. 2013, 309, 19-27. [CrossRef]

29. Gałka, B.; Kabała, C.; Łabaz, B.; Bogacz, A. Influence of stands with diversed share of Norway spruce in species structure on soils of various forest habitats in the Stołowe Mountains. Sylwan 2014, 158, 684-694.

30. Gruba, P.; Mulder, J. Tree species affect cation exchange capacity (CEC) and cation binding properties of organic matter in acid forest soils. Sci. Total Environ. 2015, 511, 655-662. [CrossRef] [PubMed]

31. Błońska, E.; Lasota, J.; Gruba, P. Effect of temperate forest tree species on soil dehydrogenase and urease activities in relation to other properties of soil derived from loess and glaciofluvial sand. Ecol. Res. 2016, 31, 655-664. [CrossRef]

32. Li, C.; Gao, S.; Zhang, J.; Zhao, L.; Wang, L. Moisture effect on soil humus characteristics in a laboratory incuba-tion experiment. Soil Water Res. 2016, 11, 37-43. [CrossRef]

33. Turunen, J.; Tomppo, E.; Tolonen, K.; Reinikainen, A. Estimating carbon accumulation rates of undrained mires in Finland-application to boreal and subarctic regions. Holocene 2002, 12, 69-80. [CrossRef]

34. Wellock, M.L.; Reidy, B.; Laperle, C.M.; Bolger, T.; Kiely, G. Soil organic carbon stocks of afforested peatlands in Irleand. Forestry 2011, 84, 441-451. [CrossRef]

35. Walz, J.; Knoblauch, C.; Böhme, L.; Pfeiffer, E.M. Regulation of soil organic matter decomposition in permafrost-affected Siberian tundra soils-Impact of oxygen availability, freezing and thawing, temperature, and labile organic matter. Soil Biol. Biochem. 2017, 110, 34-43. [CrossRef]

36. Mikutta, R.; Kleber, M.; Torn, M.S.; Reinhold, J. Stabilization of Soil Organic Matter: Association with Minerals or Chemical Recalcitrance? Biogeochemistry 2006, 77, 25. [CrossRef] 
37. Błońska, E. Effect of Stand Species Composition on the Enzyme Activity and Organic Matter Stabilization in Forest Soil; Scientific Papers of University of Agriculture in Krakow No. 527; University of Agriculture in Krakow: Kraków, Poland, 2015; Volume 404.

38. Błońska, E.; Lasota, J.; Zwydak, M.; Piaszczyk, W. Stand mixing effect on enzyme activity and other soil properties. Soil Sci. Ann. 2016, 67, 173-178. [CrossRef]

39. Osono, T.; Ono, Y.; Takeda, H. Fungal ingrowth on forest floor and decomposing needle litter of Chamaecyparis obtusa in relation to resource availability and moisture condition. Soil Biol. Biochem. 2003, 35, 1423-1431. [CrossRef]

40. Jurgensen, M.; Ree, D.; Page-Dumroese, D.; Laks, P.; Collins, A.; Mroz, G.; Degórski, M. Wood strength loss as a measure of decomposition in northern forest mineral soil. Eur. J. Soil Biol. 2006, 42, 23-31. [CrossRef]

41. Rousk, J.; Bååth, E. Growth of saprotrophic fungi and bacteria in soil. FEMS Microbiol. Ecol. 2011, 78, 17-30. [CrossRef] [PubMed]

42. Tavares, R.L.M.; Nahas, E. Humic fractions of forest, pasture and maize crop soils resulting from microbial activity. Braz. J. Microbial. 2014, 45, 963-969. [CrossRef]

(C) 2017 by the authors. Licensee MDPI, Basel, Switzerland. This article is an open access article distributed under the terms and conditions of the Creative Commons Attribution (CC BY) license (http://creativecommons.org/licenses/by/4.0/). 